\title{
CONVEX CURVES OF BOUNDED TYPE
}

\author{
A. W. GOODMAN \\ Mathematics Department \\ University of South Florida \\ Tampa, Florida 33620 \\ (Received November 30, 1984)
}

ABSTRACT. Let $C$ be a simple closed convex curve in the plane for which the radius of curvature $\rho$ is a continuous function of the arc length. Such a curve is called a convex curve of bounded type, if $\rho$ lies between two fixed positive bounds. Here we give a new and simpler proof of Blaschke's Rolling Theorem. We prove one new theorem and suggest a number of open problems.

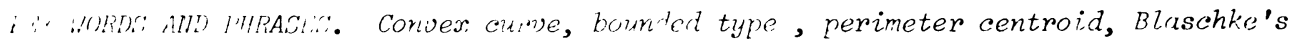
lintling Theorem, paratiol cumes, mass distribution on a curve.

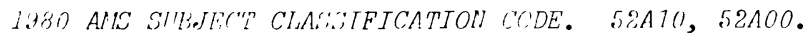

1. INTRODUCTION.

Let $\mathrm{C}$ be a simple closed convex curve in the plane. Such curves have been the subject of numerous studies $[1,2,3,5,6,7,8,12,14,15,19$, and 24] to cite only a few. Here we will refine the objects of study by looking at certain subsets. Throughout this paper $C$ is a simple closed convex curve in the plane for which the radius of curvature $\rho$ is a continuous function of arc length. Our refinement consists of putting upper and lower bounds on $\rho$.

Definition 1. We say that $\mathrm{C}$ is a convex curve of bounded type if there are constants $R_{1}$ and $R_{2}$ such that

$$
0<R_{1} \leqq \rho \leqq R_{2}
$$

at every point of $C$. We let $C V\left(R_{1}, R_{2}\right)$ denote the set of all such curves that satisfy (1.1) for fixed $R_{1}$ and $R_{2}$.

Theorems about the class $C V\left(R_{1}, R_{2}\right)$ appear in the literature (see for example Theorem 3), but as far as I am aware, this class has not been given a specific name and symbol until now. In this work we are concerned with one type of question, namely how close can C come to its "center" and how far away from its "center" can C go.

The center can be defined in various ways. For example the center of mass of the region bounded by $C$ when the region has a uniform mass distribution. Or the center could be the center of mass of the curve $C$ when the mass is distributed either uniformly or as some other function of $s$ the arc length on $C$. In any case we can take the origin as the center of mass without loss of generality. For each fixed curve in $\operatorname{CV}\left(R_{1}, R_{2}\right)$ set

$$
\mathrm{D}_{1}=\min _{\mathrm{P} \varepsilon \mathrm{C}}|\mathrm{OP}| \text {, and } \mathrm{D}_{2}=\max _{\mathrm{P} \varepsilon \mathrm{C}}|\mathrm{OP}| \text {. }
$$


Our main result is

Theorem 1. Suppose that $C \in C V\left(R_{1}, R_{2}\right)$ and the center is the center of mass of the curve $C$. If the mass distribution on $C$ is uniform, then

$$
\mathrm{R}_{1} \leqq \mathrm{I}_{1} \leqq \mathrm{D}_{2} \leqq \mathrm{R}_{2} \text {. }
$$

The two circles of radius $R_{1}$ and $R_{2}$ show that the inequality (1.3) is sharp.

Bose and Roy [6] call this center the perimeter centroid.

In section 2 we review some facts about parallel curves and we give a new proof of Blaschke's Rolling Theorem [3, pp. 114-116]. In section 3 we prove Theorem 1 . In section 4 we suggest some topics for further research on the set $C V\left(R_{1}, R_{2}\right)$.

2. PARALlel CURVES.

Let $C \in C V\left(R_{1}, R_{2}\right)$. We select the parameter $s$ (arc length) so that $s$ increases as the point $P=P(s)$ traverses $C$ in the counterclockwise direction. Let $\phi$ denote, as usual, the angle that the unit tangent $\underline{T}$ makes with the positive $x$-axis, and let $\underline{N}$ be the unit inward normal to $C$ at the point $P=P(s)$. We recall that

$$
\begin{array}{ll}
\frac{\mathrm{dx}}{\mathrm{d} s}=\cos \phi, & \frac{\mathrm{dy}}{\mathrm{ds}}=\sin \phi, \\
\underline{\mathrm{T}}=\frac{\mathrm{dx}}{\mathrm{ds}} \underline{\mathrm{i}}+\frac{\mathrm{dy}}{\mathrm{ds}} \underline{j}=(\cos \phi) \underline{\mathrm{i}}+(\sin \phi) \underline{j},
\end{array}
$$

and

$$
\underline{N}=(-\sin \phi) \underline{i}+(\cos \phi) \underline{j} .
$$

If $\underline{V}=\underline{V}(s)$ is the vector equation of $C$ we introduce a second curve $C *$ defined by the vector equation $\underline{V} *=\underline{V}(s)+A N$, where $A$ is a constant. The curve $C^{*}$ is said to be parallel to $C$, see $[13 \mathrm{pp} .80-84,18 \mathrm{p} .67$, and 19]. Fig. 1 shows a number of curves paralle1 to the ellipse $x^{2} / 9+y^{2} / 4=1$. The curve $C *$ is also a Bertrand mate of $C$, although the term Bertrand curve usually refers to twisted curves in space [4, p. 35].

If $P(x, y)$ is a point on $C$ and $P^{\star}\left(x^{\star}, y^{*}\right)$ is the corresponding point on the parallel curve $C^{\star}$, then

$$
x^{*}=x-A \sin \phi \text { and } y^{*}=y+A \cos \phi .
$$

If $k=1 / \rho$ is the curvature of $C$ at $P$, then $\kappa=d \phi / d s$ and from $(2.4)$ and $(2.1)$

$$
\frac{d x}{d s}=\frac{d x}{d s}-A \kappa \cos \phi=(1-A K) \cos \phi \text {, }
$$

and

$$
\frac{d y}{d s}=\frac{d y}{d s}-A K \sin \phi=(1-A K) \sin \phi .
$$

We let $s^{*}, k^{*}$, and $\rho^{*}$ denote, arc length, curvature, and radius of curvature at the corresponding point on $C^{\star}$. Then (2.5) and (2.6) give

$$
\left(\frac{\mathrm{ds} *}{d s}\right)^{2}=\left(\frac{d x^{*}}{d s}\right)^{2}+\left(\frac{d y *}{d s}\right)^{2}=(1-A K)^{2} \text {. }
$$

If $R_{1}<A<R_{2}$, then the curve $C^{*}$ may have cusps as shown in Fig. 1 . If $A<R_{1}$ we set $d s^{*} / d s=1-A K>0$. If $A>R_{2}$ then $1-A K<0$ and we set $d s * / d s=|1-A K|$. Thus in either case $s^{*}$ and $s$ increase together. In the first case, $A<R_{1}$, we have

$$
\begin{aligned}
\underline{T}^{*} & \equiv \frac{\mathrm{dV}}{d a^{*}}=\frac{\mathrm{dV}}{\mathrm{ds}} \frac{\mathrm{ds}}{\mathrm{ds} *}=[(1-\mathrm{AK}) \cos \phi \underline{i}+(1-\mathrm{AK}) \sin \phi \underline{j}] \frac{1}{1-\mathrm{AK}} \\
& =(\cos \phi) \underline{i}+(\sin \phi) \underline{\mathrm{j}}=\underline{\mathrm{T}} .
\end{aligned}
$$

If $A>R_{2}$, then the same type computation gives $\underline{T}^{*}=-\underline{T}$.

Lemma 1. If $A<R_{1}$, then the directed tangents at corresponding points of $C$ and $C \star$ are parallel and point in the same direction. Further $\underline{N} *=\underline{N}$. If $A-R_{2}$, then $\underline{T}^{\star}$ $=-\underline{T}$ and $\underline{N} *=-\underline{N}$. 


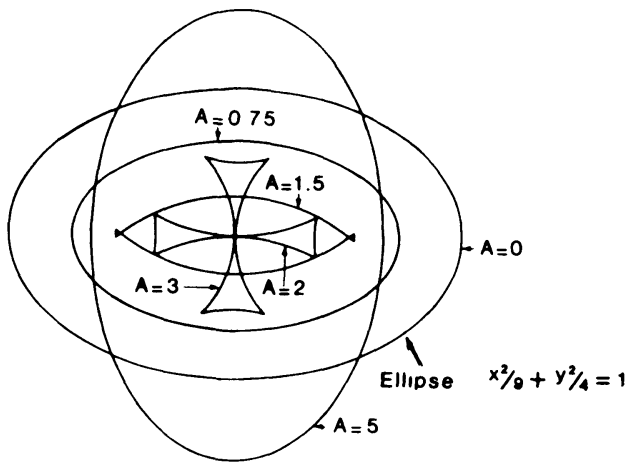

Figure 1

Lemma 2. If $C \in C V\left(R_{1}, R_{2}\right)$ and $A<R_{1}$, then $C *$ is locally convex and at corresponding points $\rho *=\rho-$ A.

By locally convex we mean $\phi^{*^{\prime}}\left(\mathrm{s}^{*}\right)>0$ at each point of $\mathrm{C}^{*}$.

Proof. From Lemma 1 we have $\phi^{*}=\phi$ at corresponding points. Hence, for the curvature

$$
\kappa^{*}=\frac{\mathrm{d} \phi^{*}}{\mathrm{ds} *}=\frac{\mathrm{d} \phi}{\mathrm{ds} *}=\frac{\mathrm{d} \phi}{\mathrm{ds}} \frac{\mathrm{ds}}{\mathrm{ds} *}=\kappa \frac{1}{1-\mathrm{A} / \rho} .
$$

Thus $\kappa^{*}>0$ whenever $\kappa>0$, and $C^{*}$ is locally convex. Further

$$
\rho *=\frac{1}{K *}=\frac{1-\mathrm{A} / \rho}{\kappa}=\rho\left(1-\frac{\mathrm{A}}{\rho}\right)=\rho-\mathrm{A} \text {. }
$$

Of course $\rho \star=\rho-A$ is geometrically obvious from the definition of C*. Q.E.D.

If $A>R_{2}$, the factor $1 /(1-A / \rho)$ in (2.9) is replaced by $\rho /(A-\rho)$. Again $C *$ is locally convex, but in this case $\rho *=A-\rho$.

It is geometrically obvious that if $A<R_{1}$ or $A>R_{2}$, then $C *$ is a simple closed curve. It seems that a direct proof is rather elusive. The difficulty may lie in the following example. Let $C *$ be the image of $|z|=1$ under the complex function $f(z)=$ $z+z^{2}$. Then $C^{*}$ is convex in the sense that $k^{\star}>0$ at every point, so $C^{*}$ is locally convex. But this curve fails to be simple. Nevertheless we have

Theorem 2. If $C \in C V\left(R_{1}, R_{2}\right)$ and $A<R_{1}$ or $A>R_{2}$, then $C *$ is a simple closed convex curve. If $A<R_{1}$, then $C * \varepsilon C V\left(R_{1} *, R_{2} *\right)$, where

$$
\mathrm{R}_{1} *=\mathrm{R}_{1}-\mathrm{A} \text {, and } \mathrm{R}_{2} *=\mathrm{R}_{2}-\mathrm{A} \text {. }
$$

If $A>R_{2}$, then $C * \varepsilon C V\left(R_{1} *, R_{2} *\right)$, where

$$
\mathrm{R}_{1} *=\mathrm{A}-\mathrm{R}_{2} \text {, and } \mathrm{R}_{2} *=\mathrm{A}-\mathrm{R}_{1} \text {. }
$$

Proof. We have already seen that $C^{*}$ is locally convex, but the example shows this is not sufficient to prove that $C^{*} \underset{\text { is }}{\text { isimple. On } C^{*} \text { let }}$

$$
\Delta \phi^{*}=\phi^{*}\left(\mathrm{~L}^{*}\right)-\phi^{*}(0)=\int_{0}^{L^{\star}} \frac{\mathrm{d} \phi^{*}}{\mathrm{ds} \mathrm{s}^{*}} \mathrm{~d} \mathrm{~s}^{*}
$$

where $L^{*}$ is the length of $C^{*}$. We make a change of variables from $s^{*}$ to $s$. If $A<R_{1}$, then $\phi^{*}=\phi$ and

$$
\frac{\mathrm{d} \phi^{*}}{\mathrm{ds}}=\frac{\mathrm{d} \phi}{\mathrm{ds}}
$$

Then (2.13) gives

$$
\Delta \phi^{*}=\int_{0}^{\mathrm{L}^{*}} \frac{\mathrm{d} \phi^{*}}{\mathrm{ds}} \frac{\mathrm{ds}}{\mathrm{ds}} \mathrm{ds}^{*}=\int_{0}^{\mathrm{L}} \frac{\mathrm{d} \phi^{*}}{\mathrm{ds}} \mathrm{ds}=\int_{0}^{\mathrm{L}} \frac{\mathrm{d} \phi}{\mathrm{ds}} \mathrm{ds}=\int_{0}^{\mathrm{L}} \mathrm{d} \phi=2 \pi .
$$


Since $C^{\star}$ is locally convex and $\Delta \phi^{*}=2 \pi$, we see that $C^{*}$ is a simple curve.

If $A>R_{2}$, then $\phi^{*}=\phi+\pi$. Hence (2.14) is still true and the proof remains valid. The relations (2.11) and (2.12) follow from $\rho *=\rho-A$ and $\rho *=A-\rho$ respectively. Q.E.D.

Theorem 3. Let $C \in C V\left(R_{1}, R_{2}\right)$ and let $K$ be a circle tangent internally to $C$ at any point $P_{0}$ of $C$. If $K$ has radius $R_{1}$, then $K$ is contained in $C$. If $K$ has radius $R_{2}$, then $K$ contains $C$.

This theorem is often called Blaschke's Rolling Theorem, because it states that (a) a circle of radius $\mathrm{R}_{1}$ can roll around the inside of $C$, and (b) a circle of radius $\mathrm{R}_{2}$ can roll around the outside of $\mathrm{C}$. Blaschke has extended his theorem to 3-dimensional space [3, p. 118]. For further work on this theorem, and various extensions see [11, 17, 20 , and 22].

To be precise the phrase "internally tangent" means that $K$ is tangent to $C$ at $P_{0}$ and the center of $\mathrm{K}$ lies on the inward normal to $\mathrm{C}$ at $\mathrm{P}_{0}$. Thus the location of the center is given by equation set (2.4) with $A$ replaced by $R_{\alpha}$ the radius of the tangent circle $(\alpha=1,2)$. We say that $K$ is contained in $C$ if $K$ is contained in the closure of the region bounded by $C$. Further $K$ contains $C$, if $C$ is in the closed disk bounded by $K$.

Proof of Theorem 3. We first show that the curve $C$ cannot cross the circle $K$ in a neighborhood of $\mathrm{P}_{0}$, the point of contact. Without loss of generality let $\mathrm{P}_{0}$ be the origin and let $K$ and $C$ be tangent to the $x$-axis at the origin. Further suppose that both the circle and the curve lie above the x-axis, except at the origin. In this position the lower half of the circle will have equation

$$
\mathrm{Y}=\mathrm{R}-\sqrt{\mathrm{R}^{2}-\mathrm{X}^{2}}, \quad-\mathrm{R} \leqq \mathrm{x} \leqq \mathrm{R} .
$$

If $y=f(x)$ is the equation of $C$ in a suitable neighborhood, $I:-\varepsilon \leqq x \leqq \varepsilon$, then we have $\mathrm{f}^{\prime}(\mathrm{x}) \operatorname{sgn} \mathrm{x} \geqq 0$ and $\mathrm{f}^{\prime \prime}(\mathrm{x}) \geq 0$ in $\mathrm{I}$.

Lemma 3. Suppose that $\rho \geq R$ in $I$, where $\rho$ is the radius of curvature on $C$. Then, under the conditions described above

$$
y(x) \leqq Y(x)=R-\sqrt{R^{2}-x^{2}}, \quad x \in I .
$$

Thus in $I$, the curve $C$ cannot cross from outside to inside $K$, but of course $C$ may coincide with $\mathrm{K}$. We omit the proof of Lemma 3, but it follows directly from two integrations, starting with the inequality

$$
\frac{1}{\rho}=\frac{y^{\prime}(x)}{\left[1+\left(y^{\prime}(x)\right)^{2}\right]^{3 / 2}} \leq \frac{1}{R} .
$$

By reversing the inequality signs we have

Lemma 4. Suppose that $\rho \leqq R$ in $I$. Then under the conditions on $K$ and $C$ described above

$$
y(x) \geq Y(x)=R-\sqrt{R^{2}-x^{2}}, \quad x \in I .
$$

Thus in $I$, the curve $C$ cannot cross from inside to outside $K$, but of course $C$ may coincide with $\mathrm{K}$.

From these two lemmas we see that if $R=R_{1}$ or $R=R_{2}$, then $C$ cannot cross into or out of $K$ in a neighborhood of a point of tangency. To complete the proof of Theorem 3 , we must obtain this same result in the large.

First suppose that $K$ has radius $R_{1}$ and is tangent internally to $C$ at $P_{0}$. If $K$ is not contained in $C$, then $K$ crosses $C$ at a point $P_{2}$ distinct from $P_{0}$. Then we may find a smaller circle $K_{0}$ with radius $R_{0}<R_{1}$, and such that $K_{0}$ is tangent internally to $C$ 
at $\mathrm{P}_{0}$, and is tangent to $\mathrm{C}$ at another point $\mathrm{P}_{1}$, see Fig. 2 .

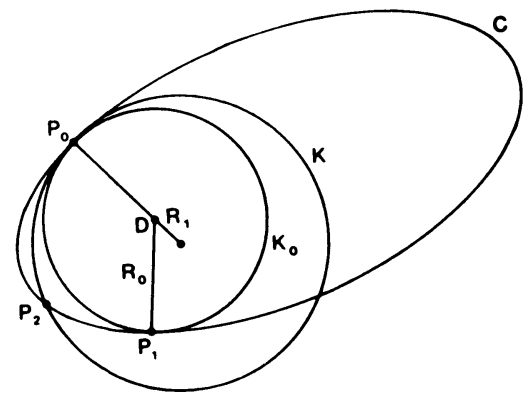

Figure 2

Now consider the parallel curve $C^{*}$ with $A=R_{0}<R_{1}$. By Theorem 2, this curve is a simple close curve. On the other hand, the center $D$ of the circle $K_{0}$ is at least a double point of $C^{\star}$ because it is the corresponding point for both $P_{0}$ and $P_{1}$. Hence we have a contradiction.

For the second part of Theorem 3 let $K$ be a circle with radius $R_{2}$ and tangent internally to $C$ at $P_{0}$. If $K$ does not contain $C$, then $K$ crosses $C$ at a point $P_{2}$ distinct from $P_{0}$. Then we may find a larger circle $K_{0}$ with radius $R_{0}>R_{2}$ and such that $K_{0}$ is tangent internally to $\mathrm{C}$ at $\mathrm{P}_{0}$ and is tangent to $\mathrm{C}$ at another point $\mathrm{P}_{1}$. Again consider the parallel curve $C \star$ with $A=R_{0}>R_{2}$. By Theorem 2 this curve $C *$ is a simple closed curve. Just as before we obtain a contradiction because $D$ the center of $K_{0}$ is at least a double point on $C \star$. Q.E.D.

Corollary 1. Let $L(C)$ denote the length of $C$ and let $A(C)$ denote the area of the region enclosed by $C$. If $C \in C V\left(R_{1}, R_{2}\right)$, then

$$
2 \pi R_{1} \leqq L(C) \leqq 2 \pi R_{2} \text {, }
$$

and

$$
\pi R_{1}^{2} \leqq A(C) \leqq \pi R_{2}^{2} \text {. }
$$

The circles of radius $R_{1}$ and $R_{2}$ show that both of these inequalities are sharp.

The inequalities (2.18) and (2.19) are well known, see for example [1, p. 352], [15], and [16, Vol. 1, pp. 529 and 548].

3. PROOF OF THEOREM 1 .

Let $C \in C V\left(R_{1}, R_{2}\right)$ and let $\mu(s)$ be a mass distribution of $C$. We exclude the trivial case in which all of the mass is concentrated at one point. Then the center of mass will be an interior point of the region bounded by $C$. Without loss of generality we select the center of mass to be the origin. If $L$ is the length of $C$, then

$$
\int_{0}^{L} x(s) \mu(s) d s=0, \quad \text { and } \quad \int_{0}^{L} y(s) \mu(s) d s=0
$$


Now consider the parallel curve $C^{*}$ where $A<R_{1}$, and let $\mu^{*}=\mu^{*}\left(s^{*}\right)$ be a mass distribution on $C^{\star}$. Then the moments $M_{x} *$ and $M_{y} *$ are given by

$$
M_{y} *=\int_{0}^{L^{*}} x *\left(s^{*}\right) \mu *\left(s^{*}\right) d s *
$$

and

$$
\mathrm{M}_{\mathrm{x}} *=\int_{0}^{\mathrm{L}} \mathrm{y*}\left(\mathrm{s}^{*}\right) \mu *\left(\mathrm{~s}^{*}\right) \mathrm{ds} \text {. }
$$

The change of variable from $s$ to $s$ yie $1 d s$

$$
M_{y} *=\int_{0}^{L}\left(x-A \frac{d y}{d s}\right) \mu *(s *(s))\left(1-\frac{A}{\rho(s)}\right) d s,
$$

and

$$
M_{x} *=\int_{0}^{L}\left(y+A \frac{d x}{d s}\right) \mu *(s *(s))\left(1-\frac{A}{\rho(s)}\right) d s .
$$

We now specialize, by setting $\mu(s)=1$ on $C$ and selecting $\mu^{\star}\left(s^{\star}\right)$ so that

$$
\mu *(s *(s))=\frac{1}{1-A / \rho(s)}>0 .
$$

Then (3.4) and (3.5) give

and

$$
M_{y} *=\int_{0}^{L}\left(x-A \frac{d y}{d s}\right) d s=\int_{0}^{L} x d s-A \int_{C} d y=0,
$$

$$
M_{x} *=\int_{0}^{L}\left(y+A \frac{d x}{d s}\right) d s=\int_{0}^{L} y d s+A \int_{C} d x=0 \text {, }
$$

from (3.1). Thus with the mass distribution (3.6), the center of mass of $C^{\star}$ is also at the origin. Since $C *$ is a simple closed convex curve, the origin lies inside $C *$ and hence $D_{1} \geq A$. Finally we note that $A$ may be taken arbitrarily close to $R_{1} \equiv$ min $|\rho|$ for points on $C$. Therefore

$$
\mathrm{D}_{1} \geq \mathrm{R}_{1} \text {. }
$$

To prove that $D_{2} \leqq R_{2}$, we consider the parallel curve $C *$ where now $A>R_{2}$. For this curve equations $(\overline{3} .2)$ and $(3.3)$ still hold. However, in this case we have

$$
\frac{\mathrm{ds} *}{\mathrm{ds}}=\frac{\mathrm{A}}{\rho}-1>0 \text {. }
$$

Thus in equations (3.4) and (3.5) we must replace the factor $1-\mathrm{A} / \rho$ by $\mathrm{A} / \rho-1$. If we

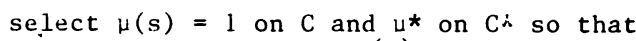

$$
\mu *(s *(s))=\frac{\rho(s)}{A-p(s)}>0,
$$

then this mass distribution will give $M_{x} *=M_{y} *=0$. By Theorem 2 , the curve $C^{*}$ is a simple closed convex curve and the origin which is also the center of mass lies inside the region bounded by $C *$.

Now let $P$ be a point on $C$ furthest from the origin. Then OP is normal to $C$ at $P$. If $P^{*}$ is the point on $C^{*}$ corresponding to $P$, then $P P^{*}$ is also normal to $C$ at $P$. Hence the points $P, 0$, and $P *$ are collinear.

Finally we observe that by Lemma 1 , the directed tangents to $C$ and $C *$ at the points $P$ and $P *$ have opposite directions. Hence the origin is an interior point of the line segment $P P^{*}$. Therefore, $|O P|<|P P *|=A$. Since A may be taken arbitrarily close to $R_{2}$, we have $D_{2} \leqq R_{2}$. Q.E.D.

4. FURTHER QUESTION FOR STUDY.

We observe that the inequality $R_{1} \leq D_{1} \leq D_{2} \leq R_{2}$ is sharp for the circles of radius $R_{1}$ and $R_{2}$. But in each extreme case $\bar{\rho}$ does not vary throughout the interval $\left[R_{1}, R_{2}\right]$ but instead is a constant at one end of the interval. The question naturally 
arises, can we find better bounds for $D_{1}$ and $D_{2} \rho$ is a continuous function of $s$ whose values fill out the interval $\left[R_{1}, R_{2}\right]$. A first candidate for consideration is the ellipse $x=a \cos t, y=b \sin t, 0 \leqq t \leqq 2 \pi$, with $0<b<a$. If we set

$$
b=\left(R_{1}{ }^{2} R_{2}\right)^{\frac{1}{3}} \text {, and } a=\left(R_{1} R_{2}{ }^{\frac{1}{3}}\right)^{\text {, }}
$$

then $\rho$ fills out the interval $\left[R_{1}, R_{2}\right]$. Further $D_{1}=b$ and $D_{2}=a$, so the expressions in (4.1) may appear as the proper lower and upper bounds for D. If true, this would improve the bounds $R_{1}$ and $R_{2}$ given in Theorem 1 . However, by piecing together arcs of circles, we can show that no better bounds than $R_{1} \leqq D_{1} \leqq D_{2} \leqq R_{2}$ can be obtained. To see this, we give $C$ only in the first quadrant and complete the curve by reflecting $C$ in the $\mathrm{x}$ - and $\mathrm{y}$-axes.

Let $C_{1}$ and $C_{2}$ be the two arcs defined by

$$
\begin{array}{ll}
x=a+R_{1} \cos t, & x=R_{2} \cos t, \\
y=R_{1} \sin t, & y=-b+R_{2} \sin t, \\
0 \leq t \leq T, & T \leqq t \leqq \pi / 2,
\end{array}
$$

respectively. The endpoints of the two arcs will meet when $t=T$ if we select $a=$ $\left(R_{2}-R_{1}\right) \cos T$ and $b=\left(R_{2}-R_{1}\right)$ sin $T$ where $0<R_{1}<R_{2}$. If we compute the first derivative for the two arcs at $t=T$, they will not be equal, but the tangent vectors will be parallel, so that for this choice of $a$ and $b$, the curve $C=C_{1} \cup C_{2}$ is a smooth curve. Further $\rho=R_{1}$ on $C_{1}$ and $\rho=R_{2}$ on $C_{2}$. Finally $D_{1}=R_{1}$ sin $T+R_{2}(1-\sin T)$ and $D_{1} \rightarrow R_{1}$ as $T \rightarrow \pi / 2$. Similarly $D_{2}=R_{2} \cos T+R_{1}(1-\cos T)$ and $D_{2} \rightarrow R_{2}$ as $T \rightarrow 0$. Thus no better bounds than $D_{2} \leqq R_{2}$ and $D_{1} \geqq R_{1}$ can be proved under the hypotheses stated. Of course $\rho$ is not continuous in a neighborhood of $P(T)$, where $C_{1}$ and $C_{2}$ meet, but it is merely a matter of labor to alter the curve slightly at $\mathrm{P}(\mathrm{T})$ to make $\rho$ continuous.

Perhaps some better bounds for $D_{1}$ and $D_{2}$ can be obtained if we impose a further restriction that the average values of $\rho$ over the curve be a fixed number such as $\left(R_{1}+R_{2}\right) / 2$. One can also examine the problem of finding sharp bounds for $D_{1}$ and $D_{2}$ if the mass distribution has some fixed pattern, other than uniform. For example, Steiner [23], and [24, pp. 99-159] has considered curves in which the mass distribution on $C$ is proportional to the curvature at each point of $C$. More generally one can select the mass distribution to be some other function of $k=1 / \rho$.

One can also consider Theorem 1, when the center of mass of $C$ is replaced by the center of mass of the region enclosed by $C$. With this replacement, Theorem 1 was proved earlier by Nikliborc [21] and Blaschke [2]. It is reasonably clear that the center of mass of a curve $C$ is in general different from the center of mass of the region enclosed by $C$, but it may be of interest to examine a particular example.

Let $C$ be $f(|z|=1)$ under $f(z)=z+a z^{2}$, where $0<a<1 / 4$. Then $C$ is symmetric with respect to the $x$-axis and if the mass distribution is uniform on $c$ then the center of mass will be on the $x$-axis. Hence it suffices to compute the $x$-coordinate. Let $\tilde{x}_{d}$ and $\tilde{x}_{C}$ denote this coordinate for the domain center of mass and the curve center of mass respectively. As easy computation gives

$$
\tilde{x}_{d}=\frac{a}{1+2 a^{2}} \text {. }
$$


A somewhat longer computation gives

$$
\tilde{x}_{C}=\frac{M_{Y}}{L} \text {, }
$$

where

$$
L=\int_{0}^{2 \pi} \sqrt{1+4 a \cos \theta+4 a^{2}} d \theta
$$

and

$$
M_{Y}=\int_{0}^{2 \pi}(\cos \theta+a \cos 2 \theta) \sqrt{1+4 a \cos \theta+4 a^{2}} d \theta
$$

Hence

$$
\tilde{x}_{C}=a\left(1-\frac{5}{2} a^{2}+\ldots\right) \text {. }
$$

It is clear that in general $\tilde{x}_{d} \neq \tilde{x}_{C}$.

We may distinguish a third center of mass $\tilde{x}_{S}$, which we will call the conformal

strip center. Suppose that $f(z)$ maps $E$ conformally onto $D$, with $f(0)=0$. Set $\tilde{x}_{s}(r, 1)$ the $x$-coordinate of the center of mass of the strip bounded by the curves $f(|z|=1)$ and $f(|z|=r)$, where $r<1$. Then by definition

$$
\tilde{x}_{s}=\lim _{r \rightarrow 1-} \tilde{x}_{s}(r, 1) \text {. }
$$

An easy computation shows that if $f(z)=z+a z^{2}, 0<a<1 / 4$, and the mass distribution is uniform, then

$$
\tilde{x}_{s}=\frac{2 a}{1+4 a^{2}}
$$

In this case $\tilde{x}_{s} \neq \tilde{x}_{d}$ unless $a=0$. Further it is clear that in general $\tilde{x}_{s} \neq \tilde{x}_{C}$. This example suggests the problem of finding

$$
\max \left|\tilde{x}_{j}-\tilde{x}_{k}\right| \text {, }
$$

when $C$ varles over the set $C V\left(R_{1} R_{2}\right)$ and $j, k \in\{d, C, s\}$.

For other relations among various centers of mass, see Guggenheimer [10], and Kubota [19].

A computation, using $|z|=1$ and

$$
\rho=\frac{\left|z f^{\prime}(z)\right|}{\operatorname{Re}\left(1+z f^{\prime \prime}(z) / f^{\prime}(z)\right)}
$$

shows that for $0 \leqq a<1 / 4$, the function $f(z)=z+a z^{2}$ gives a convex curve $f(|z|=1)$ for which the radius of curvature is

$$
\rho=\frac{\left(1+4 a \cos \theta+4 a^{2}\right)^{3 / 2}}{1+6 a \cos \theta+8 a^{2}} \text {. }
$$

Eistreme values of $\rho$ occur when $\theta=0, \theta=\pi$, and $\cos \theta=2 a$. Thus $z+a z^{2}$ is in $C V\left(R_{1}, R_{2}\right)$ for

$$
R_{1}=\sqrt{1-4 a^{2}} \text {, and } \quad R_{2}=(1-2 a)^{2} /(1-4 a) .
$$

One can also investigate the properties of normalized univalent functions that map the unit disk conformally onto a region bounded by a curve in $\operatorname{CV}\left(R_{1}, R_{2}\right)$. Some elementary results in this direction have been obtained by the author [9].

\section{REFERENCES}

1. BALL, N. HANSEN On ovals, Amer. Math. Monthly vol. 37 (1930) pp. 348-353. 2. BLASCHKE, WILHELM Über die Schwerpunkte von Eibereichen, Math. Ze1t. vol. $\underline{36}$
(1933) p. 166 . 
3. BLASCHKE, WILHELM Kreis und Kuge1, Walter de Gruyter and Co., Berlin, 1956.

4. BLASCHKE, WILHELM Differential Geometrie vols. I and II, Chelsea Publishing Co., New York, 1967.

5. BONNESEN, T. and FENCHEL, W. Theorie der Konvexen Körper, Chelsea Publishing Co., 1948 New York, N.Y.

6. BOSE, R.C. and ROY, S.N. Some properties of the convex oval with reference to its perimeter centroid, Bull. Calcutta Math Soc. Vol. 27 (1935) pp. 79-86.

7. CARATHÉODORY, C. Die Kurven mit beschrankten Biegungen, Sitz. der Preuss. Akad. Wiss. Phy. Math. Klasse (1933) pp. 102-125, Collected Works pp. 65-92.

8. FLANDERS, HARLEY A proof of Minkowski's inequality for convex curves, Amer. Math. Monthly vol. 75 (1968) pp. 581-593.

9. GOODMAN, A.W. Convex functions of bounded type Proc. Amer. Math. Soc., Vol. $\underline{92}$ (1984) Pp. 541-546.

10. GUGGENHEIMER, H. Does there exist a "Four normals triangle"? Amer. Math. Monthly vol. 77 (1970) pp. 177-179.

11. GUGGENHEIMER, H. On plane Minkowski geometry, Geometrica Dedicata vol. $\underline{12}$ (1982) pP. $371-381$.

12. GUGGENHEIMER, H. Global and local convexity, Robert E. Krieger Publishing Co. Inc. 1977 Huntington, N.Y.

13. GUGENHEIMER, H. Differential Geometry, Dover Publications 2nd edition 1977.

14. HAYASHI, T. On Steiner's Curvature-Centroid, Science Reports Tôhoku University vol. 13 (1924) pp. 109-132.

15. HAYASHI, T. Some geometrical applications of Fourier series, Circolo Mat. Palermo, Rendiconti, vol. 50 (1926) pp. 96-102.

16. HURWITZ, A. Sur quelques applications géométriques des séries de Fourier, Annales de 1'Ecole Normale supérieure vol. 19 (1902) pp. 357-408, Mathematische Werke vol. 1 pp. 510-554.

17. KOUTROUFIOTIS, DIMITRI On Blaschke's rolling theorems, Archiv der Math. vol. $\underline{23}$ (1972) pp. 655-660.

18. KREYSZIG, ERWIN O. Differential Geometry, 1959 University of Toronto Press.

19. KUВOTA, TADAHIKO Über die Schwerpunkte der konvexen geschlossenen Kurven und Flächen, Tôhoku Math. Jour. vol. 14 (1918) pp. 20-27.

20. MUKHOPADHYAYA, S. Circles incident on an oval of undefined curvature, Tôhoku Math. Jour. vol. 34 (1931) pp. 115-129.

21. NIKLIBORC, WLADYSLAW Über die Lage des Schwerpunktes eines ebenen konvexen Bereiches und die Extrema des logarithmischen Flächenpotentials eines Konvexen Bereiches, Math. Zeit. vol. 36 (1933) pp. 161-165.

22. RAUCH, JEFFERY An inclusion theorem for ovaloids with comparable second fundamental form, Journ. Diff. Geometry, vol. 9 (1974) pp. 501-505.

23. STEINER, JACOB Von dem Krummungsschwerpunkte ebener curve, Jour. reine angew. Mat. vol. 21 (1840) pp. 33-63 and 101-103.

24. STEINER, JACOB Gesammelte-Werke, Vol II Chelsea Publishing Co., New York, N.Y. 1971. 


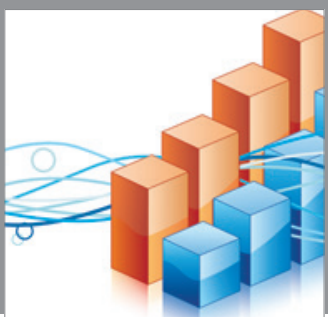

Advances in

Operations Research

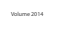

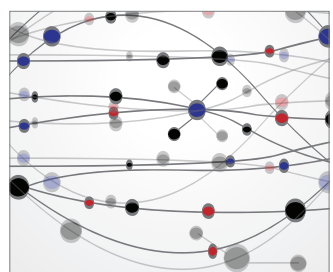

\section{The Scientific} World Journal
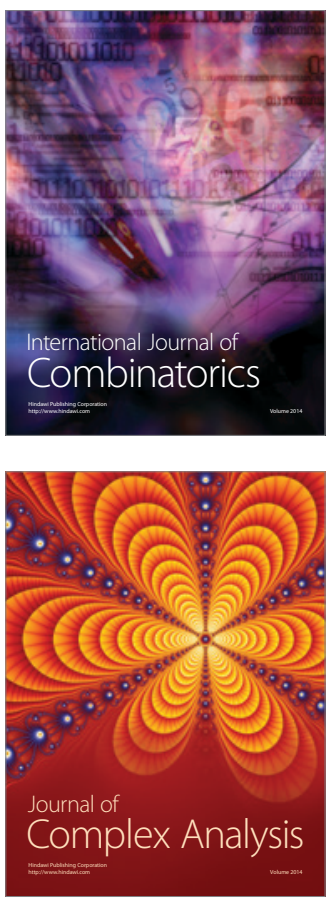

International Journal of

Mathematics and

Mathematical

Sciences
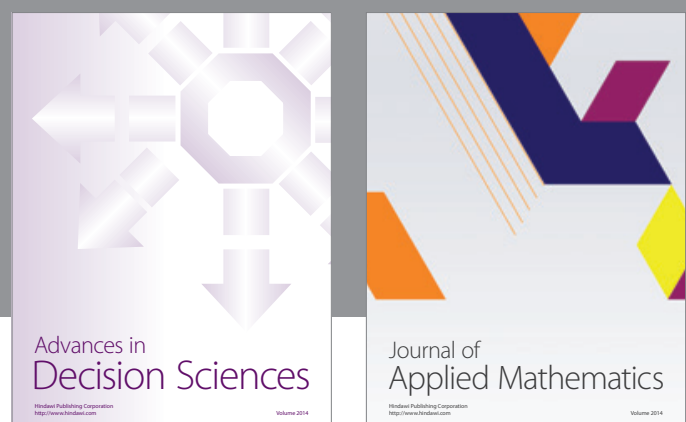

Journal of

Applied Mathematics
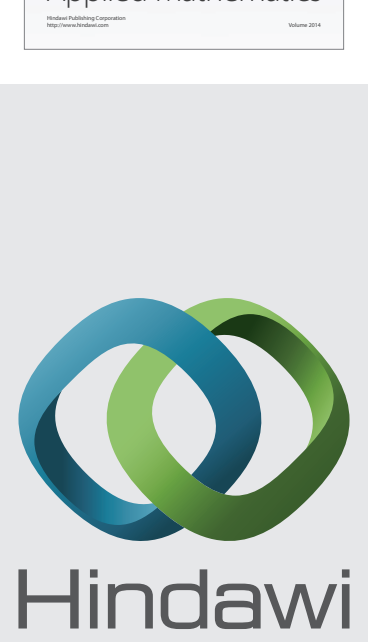

Submit your manuscripts at http://www.hindawi.com
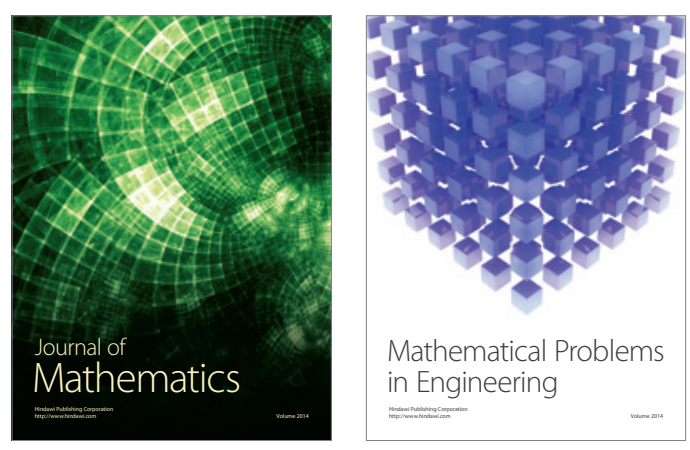

Mathematical Problems in Engineering
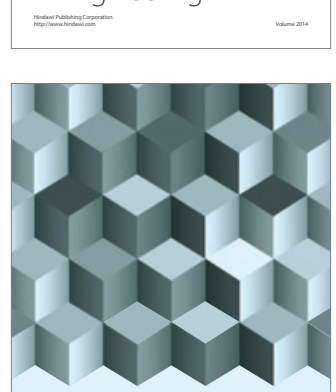

Journal of

Function Spaces
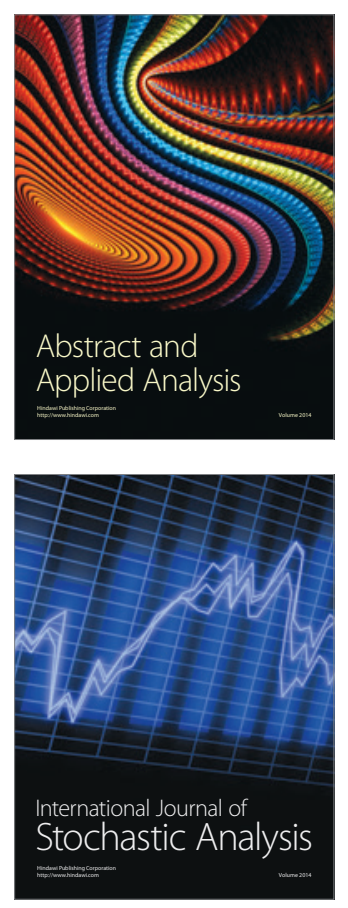

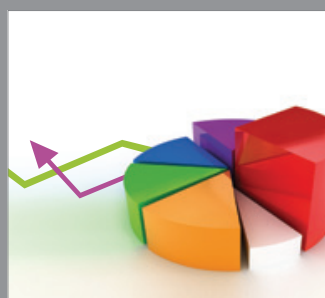

ournal of

Probability and Statistics

Promensencen
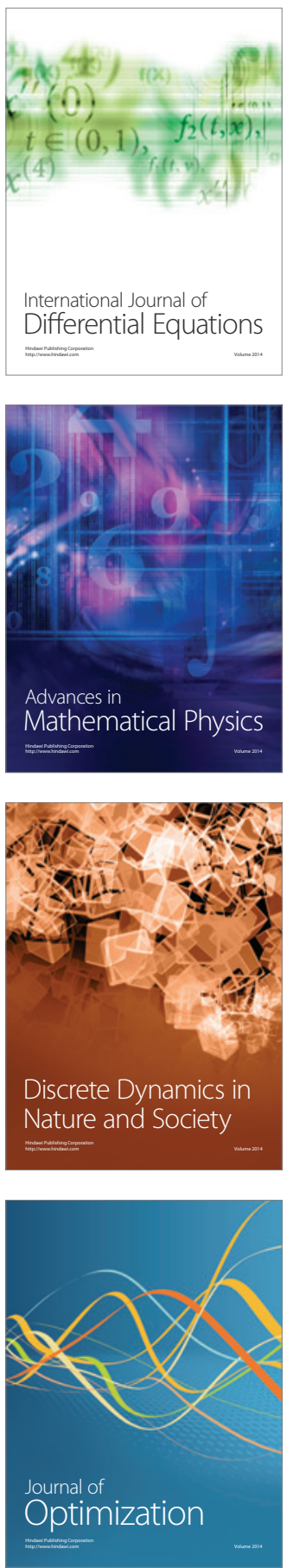